\title{
Valve Repair Using Autologous Pericardium in Tricuspid Valve Endocarditis
}

\author{
Kuldeep Singh, Ming-Li Wang, Sean A. Marco, David D’Alessandro \\ Division of Cardiothoracic Surgery, Montefiore Medical Center/Albert Einstein College of Medicine, New York, USA \\ Email: kgoraya@yahoo.com
}

Received January 5, 2012; revised February 13, 2012; accepted March 13, 2012

\begin{abstract}
Tricuspid valve endocarditis is a significant cause of valve-related morbidity and mortality. Prosthetic valve reconstruction can be costly and requires life-long anticoagulation. We present our experience with surgical management of an endocarditis damaged tricuspid valve leaflet in an intravenous drug user. We were able to excise the infected tissue and successfully repair the native valve using autologous pericardium, effectively eliminating the need for prostheses and associated complications. Our case report demonstrates that tricuspid valve reconstruction, using autologous pericardium, is a suitable option in patients with tricuspid endocarditis.
\end{abstract}

Keywords: Cardiac; Endocarditis; Heart Valve, Autograft; Pericardium; Tricuspid Valve

\section{Introduction}

Tricuspid endocarditis has increased in frequency during the last two decades, largely due to a growing number of patients addicted to intravenous drugs and to increased use of long-term central venous catheters [1,2]. Tricuspid valve endocarditis may occur in patients with congenital heart disease or may occur as a community acquired phenomenon, with no known predisposing factors [3]. When medical treatment fails to manage the infection, valve repair is an attractive option because it has been shown to be associated with lower perioperative risk than complete tricuspid valve replacement [4]. We describe our experience with a technique of using autologous pericardium to reconstruct the septal leaflet of a tricuspid valve damaged by endocarditis.

\section{Case Report}

A 40-year-old female, with history of intravenous drug use, was admitted with methicillin resistant staph aureus (MRSA) sepsis. An echocardiogram revealed large vegetations on the tricuspid valve. Despite intravenous antibiotics, the patient remained febrile and bacteremic. A subsequent CT scan of the chest revealed multiple septic emboli to bilateral lungs Figure 1. The decision was made to proceed with an operation because of failed medical therapy.

\section{Method}

The operative approach was through a median sternotomy.
Transesophageal echocardiogram (TEE) revealed normal biventricular function, large vegetation on the septal leaflet with severe regurgitation. The anterior and poster leaflets were noted to be normal. The septal leaflet was excised and reconstructed with autologous pericardium. The pericardium was soaked in glutaraldehyde, cut to size and secured to the septal edge with 5-0 proline suture Figure 2.

Two Neo chordaes were constructed with gortex sutures secured to the papillary muscle using pledgets. The height of the leaflet was based on the height of the anterior and posterior leaflets. The valve was tested for competence by injecting cold saline into the right ventricle. The valve coapted well with minimal regurgitation Figure 3.

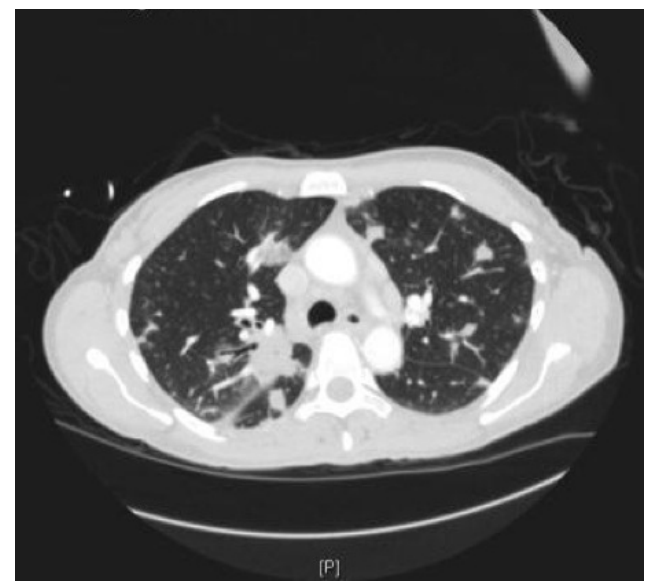

Figure 1. CT scan showing bilateral septic pulmonary emboli. 


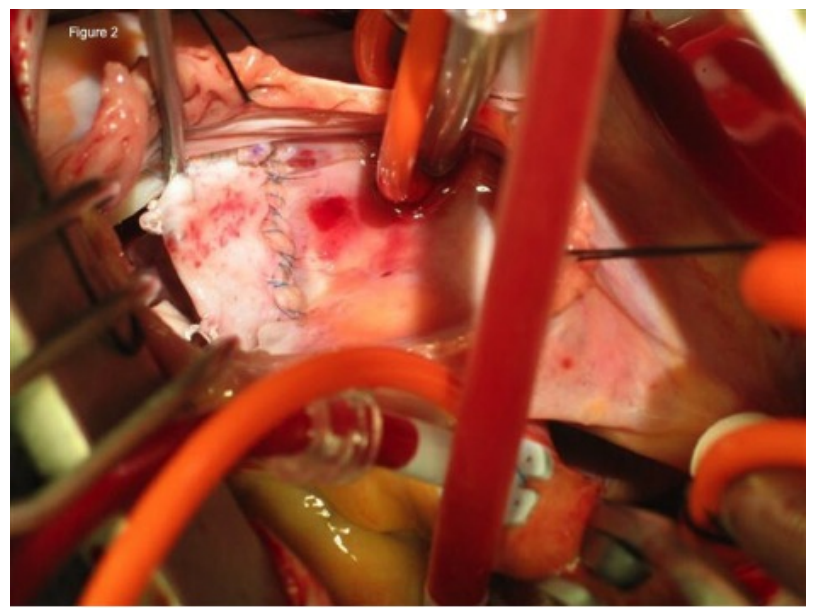

Figure 2. Septal leaflet reconstructed using autologus pericardium and secured to annulus with 5-0 proline sutures.

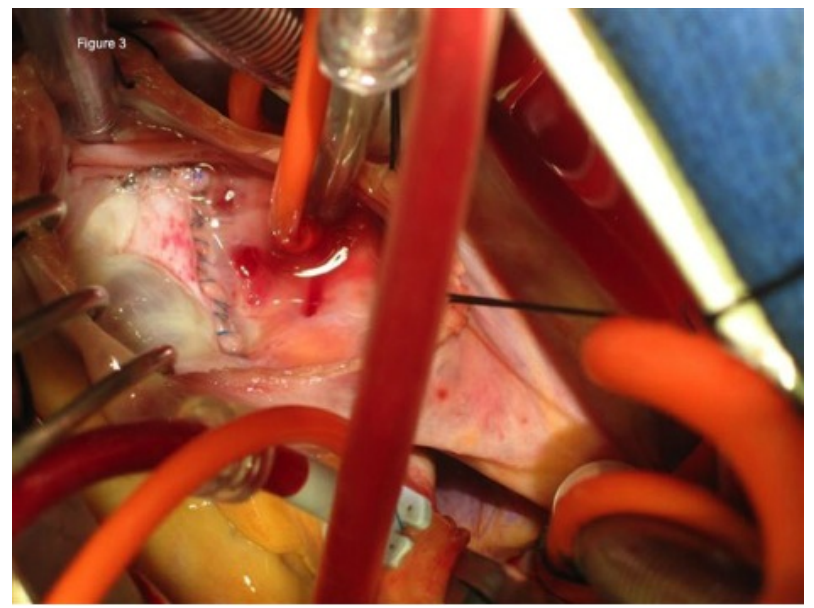

Figure 3. Full coaptation of all three valves after saline infusion of saline into the right ventricle.

Intraoperative Transesophageal echocardiogram (TEE) showed mild regurgitation, improved from severe. Parenteral antibiotic therapy was recommended for 6 weeks postoperatively. The patient was discharged from the hospital on postoperative day 16 and was lost to follow up.

\section{Discussion}

Since the first surgical treatment of tricuspid endocarditis in 1961, many options have become available, including valve replacement with prosthesis and reconstruction of the tricuspid annulus by multiple techniques. Arbulu et al. (1993) reported surgical treatment of intractable rightsided endocarditis in 55 heroin addicts via complete valve excision without valve replacement. In their follow up over 13 years, the authors observed 16 (29\%) deaths (2 progressive heart failure, 5 from uncontrollable infection, 9 from drug addiction), and 39 (67\%) survived (2 patients required a prosthetic valve for progressive right heart failure) [2].
Prosthetic valve replacement is typically the course for patients with pathologic anatomy of the tricuspid valve that excludes repair as an option. Mechanical valves have a high incidence of valve-related complications including re-infection, heart block, poor hemodynamic performance, and prosthetic thrombosis, with requirement for prolonged anticoagulation. The advent of bioprosthetic porcine valves greatly improved the prognosis after tricuspid valve replacement, as these synthetic valves do not require anticoagulation and have increased resistance to infection with lower rate of thrombosis and slower calcific degeneration [5]. Despite these advantages to the synthetic valves, little data exists on their long term durability [5]. No advantage has been demonstrated in bioprosthetic valves over mechanical valves regarding survival or re-operation rate over a 10-year follow-up [6]. There is evidence, however, for poor long-term outcome after biosynthetic replacement of the tricuspid valve after failed tricuspid repair [6].

The best short-term solution to tricuspid valve endocarditis appears to be generous excision of affected valvular tissue and reconstruction of the native valve, without the use of prostheses. For this repair to be feasible, the annulus and surrounding myocardium must be free of infection. Based upon its numerous advantages, autologous pericardium is generally considered the first choice for cardiovascular implantation [7]. Autologous pericardium is free of antigenicity, does not require permanent anticoagulation and is readily available, in addition to being cost-free. Liu et al. (2009) developed this alternative surgical approach for partial tricuspid replacement using fresh autologous pericardium in eight pigs. All surviving animals had functional, repaired tricuspid valve on echocardiography with no progression of tricuspid regurgitation and no evidence of calcification or shrinkage of the pericardial valve at 6 months after the operation [8]. We reasonably demonstrated that this approach can be applied successfully to the replacement of the entire leaflet, as long as the remaining valve is largely intact. In our case described, valve function appeared to be normal in the immediate post-operative period; however, the patient was lost to follow up. A lack of compliance and follow up is common in this difficult patient population, thus the surgeon must understand the importance of using autologous tissue use for valve repair when feasible.

\section{Conclusion}

The successful surgical treatment of tricuspid endocarditis includes the removal of all infected tissue and the restoration of valvular competence. In our case, we were able to successfully treat tricuspid endocarditis with excision of the infected tissue and with repair of the native valve using autologous pericardium. The use of autolo- 
gous pericardium to reconstruct a valve can avoid limitations of other surgical techniques, such as thrombotic complications secondary to synthetic valves, tissue antigenicity, mismatch in configuration of prosthetic valve substitutes and complications related to permanent anticoagulation. Our case report demonstrates that the technique of tricuspid valve reconstruction using glutaraldehyde treated autologous pericardium is a feasible option in patients with tricuspid endocarditis.

\section{REFERENCES}

[1] P. Chan, J. D. Ogilby and B. Segal, "Tricuspid Valve Endocarditis,” American Heart Journal, Vol. 117, No. 5, 1989, pp. 1140-1146. doi:10.1016/0002-8703(89)90874-0

[2] A. Arbulu, R. J. Holmes and I. Asfaw, "Surgical Treatment of Intractable Right-Sided Infective Endocarditis in Drug Addicts: 25 Years Experience," Journal of Heart Valve Disease, Vol. 2, No. 2, 1993, pp. 129-139.

[3] D. De La Zerda, O. Cohen, D. Marelli, F. Esmailian, D. Hekmat and H. Laks, "Tricuspid Valve Repair Using Autologous Pericardium Annuloplasty in Adults," The
Heart Surgery Forum, Vol. 11, No. 1, 2008, pp. E4-E8.

[4] P. M. McCarthy, S. K. Bhundia, J. Rajeswaran, et al., "Tricuspid Valve Repair: Durability and Risk Factors for Failure," The Journal of Thoracic and Cardiovascular Surgery, Vol. 127, No. 3, 2004, pp. 674-685. doi:10.1016/j.jtcvs.2003.11.019

[5] H. J. Stern, D. A. Sisto, J. A. Strom, R. Soeiro, S. R. Jones and R. W. Frater, "Immediate Tricuspid Valve Replacement for Endocarditis. Indications and Results,” The Journal of Thoracic and Cardiovascular Surgery, Vol. 91, 1986, pp. 163-167.

[6] F. Filsoufi, A. C. Anyanwu, S. P. Salzberg, T. Frankel, L. H. Cohn and D. H. Adams, "Long-Term Outcomes of Tricuspid Valve Replacement in the Current Era,” The Annals of Thoracic Surgery, Vol. 80, No. 3, 2005, pp. 845-850. doi:10.1016/j.athoracsur.2004.12.019

[7] R. Scrofani, M. Salati, P. Di Biasi, et al., "The Role of Autologous Pericardium in Cardiac Surgery,” The Annals of Thoracic Surgery, Vol. 63, No. 1, 1997, p. 291.

[8] D. Liu, M. Zhang and B. Song, “An Alternative Technique for Tricuspid Valve Repair Using Autologous Pericardium,” Journal of Cardiac Surgery, Vol. 24, No. 5, 2009, pp. 518-521. doi:10.1111/j.1540-8191.2009.00854.X 